\title{
O AGES AND STAGES QUESTIONNAIRES (ASQ-BR) E AÇÕES COLABORATIVAS ENTRE PAIS E EDUCADORES
}

\section{EL AGES AND STAGES QUESTIONNAIRES (ASQ-BR) Y ACCIONES COLABORATIVAS ENTRE PADRES Y EDUCADORES}

\author{
THE AGES AND STAGES QUESTIONNAIRES (ASQ-BR) AND \\ COLLABORATIVE ACTIONS AMONG PARENTS AND EDUCATORS
}

\author{
Patrícia Carla de Souza DELLA BARBA ${ }^{1}$ \\ Mayara Soler Ramos MAZAK ${ }^{2}$ \\ Evellin Eri MIYAMOTO ${ }^{3}$ \\ Maysa Marinho Antunes RAMOS ${ }^{4}$
}

RESUMO: No Brasil, percebe-se uma carência de instrumentos de monitoramento do desenvolvimento infantil que subsidiem a Intervenção Precoce. Isto posto, o Ages and Stages Questionnaires surge como uma possibilidade, visto que permite a participação de pais e educadores no acompanhamento do desenvolvimento infantil. O objetivo deste estudo foi verificar a aplicabilidade do ASQ-BR por educadores, bem como o envolvimento dos pais nesse processo. Para a coleta de dados utilizou-se o $A S Q-B R$, um pré e pós-teste aplicados aos educadores, um guia de questões para analisar suas impressões pessoais e um diário de campo para o acompanhamento da aplicação do instrumento. Os resultados revelaram que os educadores possuem capacidades para aplicar o ASQ-BR e que o instrumento permitiu maior compartilhamento de informações sobre o desenvolvimento infantil entre pais e educadores. O ASQ-BR aponta potencialidades no contexto da educação infantil, pois é um instrumento que permite acompanhar o desenvolvimento.

PALAVRAS-CHAVE: Monitoramento. Desenvolvimento infantil. ASQ-BR. Educação infantil. Família.

RESUMEN: En Brasil, hay una percepción de falta de supervisión de las herramientas de desarrollo infantil que apoyan la intervención temprana. Dicho esto, Ages and Stages Questionnaires surge como una posibilidad, ya que permite la participación de los padres y educadores en la vigilancia de desarrollo del niño. El objetivo de este estudio fue verificar la aplicabilidad de la ASQ-BR por los educadores, así como la participación de los padres en este proceso. Para la recolección de datos se utilizó el

1. Universidade Federal de São Carlos (UFSCAr), São Carlos - SP - Brasil. Profa. Dra. do Departamento de Terapia Ocupacional/PPGTO. E-mail: patriciabarba@ufscar.br

2 Universidade Federal de São Carlos (UFSCAr), São Carlos - SP - Brasil. Terapeuta Ocupacional. Email: mayaramazak@gmail.com

3 Universidade Federal de São Carlos (UFSCAr), São Carlos - SP - Brasil. Terapeuta Ocupacional. Email: evellin.miyamoto@gmail.com

4 Universidade Federal de São Carlos (UFSCAr), São Carlos - SP - Brasil. Mestranda em Terapia Ocupacional. ORCID: <http://orcid.org/0000-0003-0158-256X>. maysamarinhoramos@gmail.com 
ASQ-BR, un pre y post-test aplicado a los educadores, una guía de preguntas para analizar sus impresiones personales y un diario para controlar la aplicación del instrumento. Los resultados revelaron que los educadores tienen capacidad para aplicar el ASQ-BR y el instrumento permitió un mayor intercambio de información sobre el desarrollo del niño entre los padres y educadores. El ASQ-BR indica potencial en el contexto de la educación infantil, ya que es una herramienta para monitorear el desarrollo.

PALABRAS CLAVE: Monitoreo. Desarrollo infantil. ASQ-BR. Educación infantil. Familia.

ABSTRACT: In Brazil, there is a lack of instruments for monitoring child development that subsidize Early Intervention. This, the Ages and Stages Questionnaires emerges as a possibility, since it allows the participation of parents and educators in the monitoring of child development. The purpose of this study was to verify the applicability of $A S Q-B R$ by educators, as well as the involvement of parents in this process. To collect data, the ASQ-BR was used, a pre and post-test applied to the educators, a question guide to analyze their personal impressions and a field diary to follow the application of the instrument. The results revealed that educators have the skills to apply the ASQ-BR and that the instrument has allowed greater sharing of information on child development among parents and educators. The ASQ-BR points out potentialities in the context of early childhood education, since it is an instrument that allows monitoring the development.

KEYWORDS: Monitoring. Child development. ASQ-BR. Child education. Family.

\section{Introdução}

O desenvolvimento é um direito fundamental de qualquer criança. Ele é produto da interação dinâmica entre fatores intrínsecos (genética) e extrínsecos (ambiente) ao sujeito, devendo ser observado, sempre que possível, dentro dos contextos naturais no qual ele ocorre. As instituições de educação infantil constituem espaços peculiares de desenvolvimento, visto que o atendimento é voltado ao cuidado e educação nos seis primeiros anos de vida (REZENDE; COSTA; PONTES, 2005).

A literatura comprova a existência de um amplo conjunto de conhecimentos científicos que constatam que os cuidados que a criança recebe na primeira infância são da maior importância para seu desenvolvimento futuro, pois a primeira infância é a fase onde as respostas são mais rápidas, amplas e intensas (DELLA BARBA, 2014, p. 2).

Condições favoráveis e consistentes desde a mais tenra idade são essenciais para um desenvolvimento saudável. Todavia, algumas crianças não dispõem de todo o apoio 
necessário. É nesse sentido que a Intervenção Precoce (IP) se torna fundamental, visto que compreende um processo sistemático de planejamento e fornecimento de serviços terapêuticos e educacionais às famílias que necessitam de auxílio para atender às necessidades de desenvolvimento de crianças pequenas (PAPALIA; OLDS; FELDMAN, 2006).

Meisels e Shonkoff ( apud GRAÇA; TEIXEIRA; LOPES; SERRANO; CAMPOS, 2010) definem IP como o conjunto de serviços prestados às crianças entre zero e cinco anos de idade, com o objetivo de promover a saúde e o bem-estar, impulsionar a emergência de competências, minimizar os atrasos no desenvolvimento infantil, remediar as incapacidades existentes ou emergentes, impedir a deterioração funcional e promover a adaptação parental e o funcionamento integral da família.

Entretanto, existe uma carência de instrumentos de medida capazes de orientar as práticas de Intervenção Precoce e de monitorar, periodicamente e sistematicamente, o desenvolvimento infantil. Assim, o instrumento Ages and Stages Questionnaires $2^{\mathrm{a}}$ Edition (ASQ-2), - a Parent-Completed Child-Monitoring System - desenvolvido por Squires, Potter e Bricker em 1995 e revisto por Bricker e Squires em 1999, é um instrumento de triagem elaborado dentro de uma abordagem centrada na família e que propõe a participação de pais, cuidadores e educadores, podendo ser aplicado nos contextos familiar e escolar. Por meio do ASQ os profissionais de educação e de saúde podem auxiliar os pais na identificação de fatores de risco ao desenvolvimento e explorar elementos que oportunizem o desenvolvimento das competências de cada criança (DELLA BARBA, 2014).

O instrumento em si é composto por 19 questionários, cada um com 30 itens divididos em cinco grandes áreas: Comunicação, Coordenação Motora Ampla, Coordenação Motora Fina, Resolução de Problemas e Pessoal-Social, mais o campo de Informações Adicionais no qual os pais podem expressar demais preocupações (CAMPOS; SQUIRES; PONTE, 2011).

A tradução e a adaptação transcultural do instrumento para o Brasil foi realizada em 2010 por Filgueiras, Pires, Maissonette e Fernandez (2013), em um trabalho pioneiro no sistema público de ensino do Rio de Janeiro. Os testes mostraram que, apesar de algumas limitações, o instrumento mostrou boas propriedades psicométricas, além de ser de fácil compreensão, exigindo o mínimo de treino para aplicação. 
O estudo de Santana, Filgueiras e Landeira-Fernandez (2015), ao buscar aperfeiçoamento do instrumento, readaptou a primeira versão, o que resultou numa melhora significativa do instrumento, porém ainda com apontamentos de lacunas.

Della Barba (2014) e, posteriormente, Della Barba, Rizzo e Serrano (2015), ao analisarem o desempenho de 100 crianças no contexto escolar por meio do ASQ-BR em um município do interior do estado de São Paulo, bem como o empoderamento e conhecimento dos pais nesse processo, concluíram que o instrumento se mostra eficiente enquanto ferramenta de rastreio e monitoramento do desenvolvimento infantil.

O estudo mais recente, realizado por Fioravante-Bastos, Filgueiras e Moura (2016), ao objetivar a avaliação do instrumento por profissionais da educação infantil, apontou algumas fragilidades na aplicação do instrumento, como, por exemplo, o tempo de aplicação relativamente extenso (30 minutos), a associação do instrumento pelos educadores a uma perspectiva biopsicológica e o fato de que talvez o instrumento devesse ser aplicado por outros profissionais como, por exemplo, psicólogos e psicopedagogos, pois observou-se a descontextualização e inadequação de alguns itens do instrumento à realidade de creches e pré-escolas.

Assim, sabendo que as instituições de educação infantil atendem por um longo período de tempo crianças de 0 a 6 anos e que os educadores assumem um papel importante para o desenvolvimento infantil, uma vez que são responsáveis pela mediação dos processos de aprendizagem resultantes da interação da criança com o ambiente, não se pode negar a potencialidade desses espaços e profissionais na identificação de fatores de risco e proteção ao desenvolvimento infantil (DELLA BARBA, 2014). Ainda, estudos apontam quão importante se fazem as trocas de informação entre os familiares e educadores no decorrer do trabalho desenvolvido com crianças.

É nesse sentido que a pesquisa ora apresentada, a fim de ampliar a investigação sobre o empoderamento de pais e educadores em prol do desenvolvimento infantil a partir do ASQ-BR, teve como objetivo identificar a aplicabilidade do instrumento ASQBR por educadores do ensino infantil em um município do interior do estado de São Paulo e a possibilidade de envolvimento dos pais nesse processo.

\section{Método}

\section{Participantes}


Participaram desta pesquisa 19 educadores que atuam em 4 Centros Municipais de Educação Infantil (CEMEIs) do município de São Carlos, bem como 90 pais e responsáveis de crianças de 28 a 66 meses de idade que frequentam as instituições.

\section{Instrumentos}

O instrumento central utilizado foi o Ages and Stages Questionnaires Brasil (ASQ-BR). Esse instrumento visa o rastreio e o monitoramento do desenvolvimento de crianças de 5 a 66 meses de idade que são observadas em cinco áreas (Comunicação, Coordenação Motora Ampla, Coordenação Motora Fina, Resolução de Problemas e Pessoal-Social), mais um campo de observações no qual os pais e os educadores podem expressar demais preocupações.

Para cada item o aplicador deve assinalar "sim" para indicar que a criança realiza determinada atividade, "às vezes" para indicar que a criança a realiza ocasionalmente e "não" para as atividades ainda não realizadas. Em seguida as respostas são convertidas em pontos $(0,5$ e 10$)$ que são totalizados por área. Caso a pontuação da criança se encontre abaixo do ponto de corte é recomendada uma avaliação mais detalhada. Se estiver entre os pontos de corte indica-se um acompanhamento da criança junto à família. E se a pontuação se encontrar acima do ponto de corte entende-se que a criança apresenta um desenvolvimento esperado. Neste estudo, tendo em vista a faixa etária selecionada, foram utilizados apenas os sete questionários que abrangem crianças dos 28 aos 66 meses de idade. Ressalta-se que, embora os estudos que estão sendo realizados vislumbrem um escore normativo para o Brasil, esse ainda não existe, sendo utilizada a nota de corte americana.

Também foram realizados um pré e pós-teste para averiguar os conhecimentos prévios e adquiridos dos educadores acerca do desenvolvimento infantil, bem como um guia de questões direcionadas para identificar as suas impressões pessoais frente à aplicação do instrumento e um diário de campo no qual os educadores puderam descrever todo o processo de aplicação do ASQ-BR, bem como do compartilhamento dos resultados com os pais e familiares envolvidos.

\section{Procedimentos}

\section{Seleção dos participantes}


Os participantes foram selecionados por intermédio da Secretaria Municipal de Educação de São Carlos, que indicou quatro instituições interessadas em participarem da pesquisa.

\section{Capacitação dos educadores}

A capacitação dos educadores para a aplicação do ASQ-BR foi realizada no Horário de Trabalho Pedagógico Coletivo (HTPC) de cada instituição, por um período de 2 horas. Para tanto, foram oferecidos um manual de uso do instrumento, bem como os sete questionários que compreendem a faixa etária analisada. Nesse momento, também foram realizados o pré e o pós-teste, além de algumas perguntas acerca das concepções do educador sobre o desenvolvimento infantil e sobre a contribuição do instrumento para a sua prática profissional.

\section{Coleta de dados}

Os dados oriundos do pré e pós-teste foram reunidos pelas pesquisadoras, no momento da capacitação. Já os dados do ASQ-BR foram coletados pelos educadores, sob supervisão das pesquisadoras, no decorrer da sua rotina normal de trabalho. Os dados do guia de questões e do diário de campo foram reunidos durante o processo de aplicação do instrumento e nos horários de entrada e saída das crianças, visto que é o momento no qual os educadores podem, com mais facilidade, trocar informações com os pais e familiares.

\section{Análise de dados}

Os dados foram organizados e analisados qualitativamente por meio da Análise de Conteúdo Temática de Bardin (2011), que consiste na descrição dos conteúdos coletados e no levantamento de indicadores e temas que permitam inferir conhecimentos.

\section{Considerações éticas}


A pesquisa, protocolada sob o número 510.353/2014, foi realizada mediante aprovação do Comitê de Ética e Pesquisa em Seres Humanos da Universidade Federal de São Carlos (UFSCar).

\section{Resultados e discussão}

Os resultados ora apresentados fazem alusão ao processo de capacitação para o uso do ASQ-BR, à aplicabilidade prática do instrumento e ao compartilhamento de informações entre pais e educadores acerca do desenvolvimento das crianças.

A repercussão da capacitação é evidenciada na melhora do desempenho dos educadores, sendo que $84,2 \%$ adquiriu conhecimento sobre os itens do instrumento, o que revela uma potencialidade do ASQ-BR frente à compreensão do desenvolvimento infantil e a necessidade de formação continuada especializada dos educadores, para que possam oferecer o cuidado e a educação adequados a essa etapa da vida, marcada por rápidas e intensas transformações.

No que se refere à aplicabilidade prática do instrumento, 91,6 \% dos educadores o considerou completo, por contemplar todas as áreas do desenvolvimento da criança, reconhecendo a sua contribuição para um entendimento mais amplo acerca das capacidades individuais de cada uma. Além disso, 80\% dos educadores acreditam que, por fornecer informações importantes sobre o desenvolvimento, o ASQ-BR tem potencial para ser utilizado no âmbito da educação infantil, auxiliando a prática pedagógica na articulação das atividades e impulsionando a colaboração entre escola e família. Contudo, o tempo demandado para a aplicação do teste destaca-se como um desafio ao processo de aplicação, visto que $100 \%$ dos educadores atentam para a dificuldade encontrada em adequar esse momento à rotina das creches e pré-escolas.

\footnotetext{
"São pequenas coisas no dia-a-dia que não damos muita importância e esse questionário nos fez refletir melhor"

"Muito importante a utilização do instrumento, com a parceria da universidade, professores, pais e alunos consegue-se um resultado positivo"
}

"Tenho segurança no meu trabalho, porém as capacitações são sempre bem-vindas e acrescentam muito" 
"O instrumento é importante para o desenvolvimento do trabalho na Educação Infantil, para nortear o trabalho pedagógico com o objetivo de melhorar o desenvolvimento da criança"

Concernente ao compartilhamento das informações entre pais e educadores acerca do desenvolvimento das crianças, constatou-se que a 37,9\% delas foi atribuída alguma dificuldade ou queixa advinda dos educadores e que foram com os pais e familiares dessas crianças que houve o estabelecimento de trocas, sugerindo que a atenção ao desenvolvimento atípico se sobrepõe ao desenvolvimento típico e impede, muitas vezes, o estabelecimento de uma rede preventiva de atenção à criança, visto que a atenção se dá quando já há algum problema instalado. Dentre os aspectos discutidos entre pais e educadores, destacaram-se: questões médicas e de saúde (35\%), dificuldades relacionadas à fala e à linguagem (29\%) e aspectos comportamentais (23\%), talvez por serem aspectos mais perceptíveis, seguidos de atenção/concentração (6\%), coordenação motora (5\%) e questões relacionadas à alimentação (1\%).

Ressalta-se, ainda, que houve retorno positivo dos pais aos educadores, porém, em contrapartida, os educadores relataram dificuldades em contatar os pais, visto que muitos trabalhavam e não conseguiam arranjar tempo para responder às questões relacionadas à rotina da criança no âmbito familiar. Por fim, os educadores informaram que, embora o compartilhamento com os pais já fizesse parte da rotina das instituições, o uso do instrumento potencializou as ações colaborativas, intensificando assim o vínculo com as famílias e o conhecimento acerca do cotidiano da criança.

\section{Considerações finais}

Consideramos que o ASQ-BR consiste em um instrumento em potencial na triagem do desenvolvimento infantil, sobretudo quando utilizado no âmbito de creches e pré-escolas, visto que por meio dele é possível monitorar o desenvolvimento global de crianças de maneira mais sistemática, periódica e articulada. A articulação entre pais e educadores na promoção de capacidades se faz cada vez mais necessária, haja vista que ambos são os principais mediadores deste processo, sendo capazes, portanto, de identificar precocemente qualquer sinal de atraso que possa vir a prejudicar o desenvolvimento posterior da criança. Além disso, é nesse espaço que a maioria das crianças, sobretudo aquelas oriundas de famílias de trabalhadores, que demandam por serviços públicos de qualidade, estão inseridas e, portanto, é nesses espaços, ocupados 
pelos que mais necessitam de apoio, para onde o olhar deve se voltar. Sendo assim, julga-se necessária a reflexão contínua acerca da formação inicial e continuada dos profissionais envolvidos no cuidado e educação de crianças de 0 a 6 anos de idade, bem como na expansão de pesquisas acerca do desenvolvimento infantil e da intervenção precoce.

Ressalta-se que somente no Brasil o ASQ-BR está sendo utilizado no contexto da educação infantil, implicando num desdobramento positivo para futuras pesquisas acerca do instrumento no país. Somado a isso, diante da carência de instrumentos de monitoramento do desenvolvimento infantil no Brasil, o ASQ-BR apresenta boas perspectivas enquanto um instrumento fácil e acessível a pais e educadores.

AGRADECIMENTOS: Ao Conselho Nacional de Desenvolvimento Científico e Tecnológico (CNPq).

\section{REFERÊNCIAS}

BARDIN, L. Análise de conteúdo. 3. reimp. Lisboa: Edições, v. 70, 2011.

DELLA BARBA, P. C. S.; RIZZO, I. C.; SERRANO, A. M. P. 2015. Ages and Stages Questionnaires, um sistema facilitador do envolvimento parental e do conhecimento do desenvolvimento infantil. Revista Portuguesa de Terapia da Fala (APTF), v. 4, p. 23 29, 2015. DOI: 10.21281/rptf.2015.04.04.

DELLA BARBA, P. C. S. O empoderamento de pais para o conhecimento sobre o desenvolvimento de seus filhos: o Ages and Stages Questionnaires Brasil - ASQ-BR. Relatório de Pesquisa CAPES (Pós-Doutorado em Estudos da Criança). Universidade do Minho, Braga, Portugal. 2014. Processo CAPES no. 10921/13-3.

FILGUEIRAS, A.; PIRES, P.; MAISSONETTE, S.; FERNANDEZ, L. Psychometric Properties of the Brazilian-Adapted Version of the Ages and Stages Questionnaire in Public Child Daycare Centers. Early Human Development, 2013. DOI: 10.1016/j.earlhumdev.2013.02.005.

FIORAVANTI-BASTOS, A. C. M.; FILGUEIRAS, A.; MOURA, M. L. S. Avaliação do Ages and Stages Questionnaires-Brasil por profissionais de Educação Infantil. Estud. Psicol.,Campinas, v. 33, n. 2, p. 293-301, jun., 2016. DOI: 10.1590/198202752016000200011.

GRAÇA, P. R.; TEIXEIRA, M. L. S. C.; LOPES. S. C. G.; SERRANO, A. M. S. P. H.; CAMPOS, A. R. S. O momento da avaliação na intervenção precoce: o envolvimento da família estudo das qualidades psicométricas do ASQ-2 dos 30 aos 60 meses. Revista Brasileira de Educação Especial, Marília, v.16, n.2, p.177-196, maio/ago., 2010. DOI: 10.1590/S1413-65382010000200003. 
PAPALIA, D. E.; OLDS, S. W.; FELDMAN, R. D. Desenvolvimento Cognitivo nos Três Primeiros Anos. In: PAPALIA, D. E.; OLDS, S. W.; FELDMAN, R. D.

Desenvolvimento humano. Ed. Artmed, 8 ed. 2006. p. 186- 226.

REZENDE, M. A.; DA SILVA COSTA, P.; PONTES, P. B. Triagem de desenvolvimento neuropsicomotor em instituições de educação infantil segundo o teste de Denver II. Escola Anna Nery Revista de Enfermagem, v. 9, n. 3, p. 348-355, 2005. DOI: $10.1590 /$ S1414-81452005000300003.

SANTANA, C. M.T.; FILGUEIRAS, A.; LANDEIRA-FERNANDEZ, J. Ages \& Stages Questionnaire-Brazil-2011 Adjustments on an Early Childhood Development Screening Measure. Global Pediatric Health, v. 2, p. 2333794X15610038, 2015. DOI: $10.1177 / 2333794 X 15610038$.

\section{Como referenciar este artigo}

DELlA BARBA, Patrícia Carla de Souza et al. O Ages and Stages Questionnaires (ASQ-BR) e ações colaborativas entre pais e educadores. Revista Temas em Educação e Saúde, Araraquara, v.14, n.1, p. 31-40, jan./jun., 2018. E-ISSN: 2526-3471. DOI: 10.26673/rtes.v14.n1.2018.10581

Submetido em: $21 / 10 / 2017$

Aprovado em: 04/05/2018 